\author{
CORPUS \\ Corpus \\ $11 \mid 2012$ \\ La cooccurrence, du fait statistique au fait textuel
}

\title{
La colligation : autre nom de la collocation grammaticale ou autre logique de la relation mutuelle entre syntaxe et sémantique?
}

Dominique Legallois

\section{(2) OpenEdition \\ Journals}

Édition électronique

URL : http://journals.openedition.org/corpus/2202

ISSN : 1765-3126

Éditeur

Bases ; corpus et langage - UMR 6039

Édition imprimée

Date de publication : 1 janvier 2012

ISSN : 1638-9808

\section{Référence électronique}

Dominique Legallois, " La colligation : autre nom de la collocation grammaticale ou autre logique de la relation mutuelle entre syntaxe et sémantique? », Corpus [En ligne], 11 | 2012, mis en ligne le 21 juin 2013, consulté le 07 septembre 2020. URL : http://journals.openedition.org/corpus/2202

(c) Tous droits réservés 


\title{
La colligation : autre nom de la collocation grammaticale ou autre logique de la relation mutuelle entre syntaxe et sémantique?
}

\author{
Dominique LEGALLOIS \\ Crisco, Université de Caen
}

\section{Introduction}

Collocation grammaticale et colligation sont-ils des termes synonymes ? Renvoient-ils exactement aux mêmes phénomènes de solidarité entre unités grammaticales? Ces questions se posent pour deux raisons au moins: premièrement, dans la mesure où on constate que de plus en plus d'articles réfèrent à la notion de colligation, et cela, en dehors même du cadre de la linguistique contextualiste britannique dans lequel la colligation a été « inventée », il paraît important d'apporter un éclairage sur ces notions. La colligation vient-elle concurrencer la collocation grammaticale, dans une sorte d'inflation terminologique, ou répond-elle à une logique autre ? Deuxièmement, discuter de la relation entre collocation grammaticale et colligation, sur la base d'éléments historiques, critiques et analytiques, permet de montrer en quoi la collocation grammaticale et la colligation constituent des phénomènes particulièrement pertinents pour les sciences du langage, puisque leur étude révèle des solidarités syntagmatiques au cœur des productions effectives mais aussi au cœur même du système linguistique. Autrement dit, il y a légitimité à considérer que ces phénomènes sont centraux pour une réflexion théorique générale en grammaire et en linguistique du texte.

Cet article est organisé ainsi : la première section discute de la notion de collocation lorsque celle-ci met en jeu des unités grammaticales ; la grammaire est-elle affectée par des relations de solidarités telles que celles que connaissent les lexèmes ? La

Corpus $n^{\circ} 11$, La cooccurrence : du fait statistique au fait textuel

(2012), 31-54 


\section{LEGALLOIS}

deuxième section présente et discute la notion de colligation, depuis les propos de son inventeur, J. Firth. Il s'agira alors de souligner les différences entre collocation et colligation et de présenter des développements récents. La troisième section s'intéresse à quelques «incarnations» des unités collocationnelles ou/et colligationnelles: des cadres collocationnels d'A. Renouf et J. Sinclair, à ces unités que constituent les " motifs" grammaticaux. Ces derniers forment une catégorie dont j'illustrerai la pertinence par un ensemble d'exemples pris dans la littérature ou travaillés par mes soins. Je présenterai en particulier quelques motifs spécifiques du genre poétique, en montrant que ces unités permettent d'observer des relations intertextuelles.

\section{La collocation grammaticale?}

\subsection{Combinaison lexicale}

Traditionnellement, on n'associe que très rarement collocation et unités grammaticales. La collocation est essentiellement, dans la pratique linguistique, une manifestation de solidarités lexicales, que la lexicologie - et non la grammaire - se doit de mettre en évidence. Si on considère les conceptions récentes de la collocation, on n'y détecte pas de places particulières qui seraient accordées aux mots "outils», ou aux grammèmes de Martinet; la définition de la collocation que proposent, par exemple, Tutin \& Grossmann (2002) - définition qui me paraît tout à fait opératoire - n'accorde pas de place aux unités grammaticales ; elle est en cela représentative de la conception dominante :

Une collocation est l'association d'une lexie (mot simple ou phrasème) $\mathbf{L}$ et d'un constituant $\mathbf{C}$ (généralement une lexie, mais parfois un syntagme, par exemple à couper au couteau dans un brouillard à couper au couteau) entretenant une relation syntaxique telle que :

- C (le collocatif) est sélectionné en production pour exprimer un sens donné en cooccurrence avec L (la base);

- le sens de L est habituel (Tutin \& Grossmann $2002: 5$ ).

$\mathrm{Au}$ niveau sémantique, il convient alors de distinguer entre collocations opaques (dont on ne peut interpréter di- 


\section{La colligation}

rectement le sens : peur bleue, colère noire), collocations transparentes (dont on ne peut prédire le sens : grièvement blessé, faim de loup), collocations régulières (le collocatif inclut le sens de la base, ou a un sens très générique : nez aquilin, grande tristesse). Cette perspective, et les exemples qui l'illustrent, indiquent clairement que la démarche adoptée est lexicologique et « lexématique ». Lexicologique, dans la mesure où les auteurs s'intéressent à la collocation comme phénomène linguistique général. On remarquera pourtant, sans qu'il y ait incompatibilité, que l'étude de la collocation peut avoir également un intérêt textométrique. Si on contraste les romans de Zola, de Hugo et de Balzac, les collocations faire fortune, faire faillite et faire banqueroute ne présentent pas le même degré d'associativité

Tableau 1. degré de collocation dans trois corpus romanesques

\begin{tabular}{|l|c|c|c|}
\hline & BALZAC & HUGO & ZOLA \\
\hline Faire fortune & 131,31 & $\varnothing$ & 21,38 \\
\hline Faire faillite & 74,96 & 14,58 & 23,27 \\
\hline Faire banqueroute & 7,99 & 5,98 & 5,91 \\
\hline
\end{tabular}

S'il y a toujours collocations (à part faire fortune chez Hugo) dans les trois corpus romanesques, le degré de signification à accorder à leurs manifestations est fort différent. Sur un plan discursif, interprétatif et comparatif, le faire fortune chez Balzac n'a pas de commune mesure avec le faire fortune de Zola.

La conception de la collocation qui apparaît dans la citation d'A. Tutin et F. Grossmann est également «lexématique ». Il convient d'entendre par cette caractérisation que la collocation est un phénomène mettant en jeu essentiellement des lexèmes et non des unités grammaticales ou fonctionnelles. Cette citation de U. Heid est en ce sens très représentative de la conception habituelle de la collocation :

\footnotetext{
collocations are combinations of exactly two lexemes (of category noun, verb, adjective or adverb), realizing two concepts where the choice of one of them depends on (or : is restricted by) the other. (U. Heid 1994 : 228, cité par A. Tutin, à par.)
} 


\section{LEGALLOIS}

Il y a cependant quelques nuances à apporter. Premièrement, se posant la question de prendre en compte les unités grammaticales dans l'identification des collocations, Tutin (à par.) propose de considérer comme seules pertinentes les prépositions non régies (par ex. de dépit), et donc, d'ignorer les associations qui relèvent de configurations syntaxiques (par ex. une préposition régie introduisant un argument: je me souviens de cela). Le risque selon moi de ce parti-pris est peut-être d'être amené à considérer ce qui relève traditionnellement de la locution comme cas de collocation. De dépit, par exemple, n'est pas habituellement jugé comme une collocation. Deuxièmement, dans l'analyse des collocations, le grammatical n'est jamais totalement évacué : d'une part, parce que la collocation est une association restreinte définie dans le cadre d'un rapport syntaxique (par ex. nom / épithète); d'autre part, parce que les généralisations à partir de patrons syntaxiques - telles ce que celles opérées par Hausmann (1989 - par ex. N+Adj., V+Adv., etc.) - font partie de l'analyse essentielle des phraséologues. Il reste que ces patrons syntaxiques ne sont pas donnés, évidemment, comme des combinaisons elles-mêmes collocationnelles. L'emploi du terme collocation grammaticale pour désigner ces patrons syntaxiques est donc trompeur.

La partie suivante montre que le phénomène de collocation n'a pas toujours été considéré comme purement lexical, mais qu'il a concerné tout type d'unités linguistiques.

\subsection{La collocation grammaticale : un bref aperçu historique}

L'acception moderne de collocation est très souvent attribué à J.R. Firth. Rien de plus inexact, puisque son collègue H. Palmer l'employa, dans son sens technique, avant lui (Palmer 1933). Mais on peut s'appuyer sur des références plus anciennes encore. Ainsi, dans un de ses emplois au $18^{\mathrm{e}}$ siècle, collocation était étroitement associé à la colligation. C'est ce que remarque S. Bartsch (2004). L'Oxford English Dictionary ( $2^{\mathrm{e}}$ éd.) donne pour collocation la citation suivante :

1750 Harris Hermes ii. iv. Wks. 197 The accusative in modern languages being subsequent to its verb, in the collocation of the words. 


\section{La colligation}

S. Bartsch note alors :

in the quotation by Harris, collocation is used in a sense that is now commonly covered by the closely related term « colligation », i.e. the grammatical juxtaposition of words in sentences. (Bartsch 2004 : 29)

Cette conception est encore présente chez O. Jespersen. Par exemple, dans Language de 1922, le mot est synonyme d'usage régulier d'une suite de formes pouvant donner lieu à des coalescences :

From the collocation in 'I have my hand full of peas' the transition is easy to 'a handful of peas' where the accentual subordination of full to hand paves the way for the combination becoming one word instead of two. (Jespersen $1922: 376$ )

Le terme s'applique donc ici aux relations syntagmatiques de mots dans le cadre de la proposition. Mais collocation peut aussi, chez Jespersen, s'employer comme suite ou ordre marqué de mots, ou, si on veut, comme organisation syntaxique particulière. On est alors dans une conception ouvertement grammaticale, non pas en termes de nature de mots, mais en termes fonctionnels de combinatoire ; dans le vers de Gray

And all the air a solemn stillness holds, [...] it does not matter much, for the ultimate understanding of the line must be exactly the same whether the air holds stillness or stillness holds the air. In ordinary language we may find similar collocations, but it is worth saying with some emphasis that there can never be any doubt as to which is the subject and which the object. (Jespersen $1922: 345$ )

Ce dernier exemple est particulièrement spectaculaire, puisqu'il met en jeu non pas des mots grammaticaux, mais des fonctions syntaxiques. On a affaire encore, comme on le verra, à un cas que Firth désignera sous le terme de colligation. Une autre occurrence de collocation chez Jespersen - la première, à ma connaissance, de la linguistique moderne - porte sur la combinaison d'unités grammaticales : 


\section{LEGALLOIS}

In English scarcely any, scarcely ever is generally preferred to the combinations almost no, almost never [...]. Little and few are also incomplete negatives: note the frequent collocation with no : there is little or no danger. (Jespersen 1917 :

$39-40)$

Le mot collocation désigne donc dans cet emploi « historique » un fait d'association entre éléments grammaticaux, association remarquée en raison de sa fréquence. Le terme désigne donc chez Jespersen autant les associations entre mots grammaticaux ou lexicaux, que les places ou l'ordre des éléments dans une proposition. Comme dit plus haut, cette dernière acception relève de la colligation.

Si on s'intéresse non plus à l'emploi du mot collocation mais à la notion d'association à laquelle il renvoie, la référence à Bally s'impose alors, puisque le linguiste genevois intègre les « faits de syntaxe » dans les unités phraséologiques :

La négation ne...que est une unité phraséologique; d'une manière générale on pourrait prouver qu'une foule de faits de syntaxe ne sont que des groupements phraséologiques à éléments séparables. (Bally 1909 : 76)

Cet exemple, encore :

La notion d'appartenance ou de propriété est exprimée en français par des groupes comme « la maison de Paul » où « maison » et «Paul» peuvent être remplacés par n'importe quel substantif, la préposition étant la partie fixe du groupe; qu'est-ce là sinon une locution à éléments interchangeables? (Bally 1909 : 77)

Si on n'est pas, avec ces exemples, dans un rapport qui s'apparenterait entièrement à une collocation lexicale, on perçoit néanmoins une conception des solidarités grammaticales qui ne doit plus rien à une quelconque compositionnalité syntaxique. Le dernier exemple de Bally est, à ce sujet, particulièrement intéressant. Sans y faire directement référence, B. Pottier, bien plus tard, proposa une conception très semblable des relations syntaxiques avec la notion de syntaxie ou de modèle syntaxique usuel : 


\section{La colligation}

«Le N1 + de + N2» (par ex. le livre de Pierre)

est un modèle primaire de langue. (Pottier 1968 :

20)

La grammaire se phraséologise à travers l'usage de régularités constructionnelles. La notion de cadre collocationnel, proposée par Renouf \& Sinclair (1991) va prolonger cette idée, comme on le verra dans la section 3 .

\section{La colligation}

Collocation grammaticale et colligation sont parfois donnés comme synonymes ou quasi synonymes (cf. Granger \& Paquot $2008: 33$, note 4 , qui néanmoins tempèrent cette équivalence par un exemple : " the word involvement is said to colligate with prepositions but to collocate with in and with »). Il me semble que la distinction entre collocation grammaticale et colligation est pourtant souhaitable, car elle renvoie à des solidarités portant sur des éléments différents.

La sous-section suivante apporte un éclairage à partir des propos - pourtant assez obscurs - de J.R. Firth.

\subsection{La colligation chez Firth et ses continuateurs}

Il est probable que J.R. Firth ait rencontré Ch. Bally lors d'un séjour à Genève dans les années 20 (Rebori 2002). On peut aimer supposer que le suisse mit alors au courant le britannique de sa conception phraséologique des « faits de syntaxe ». Evidemment, aucune source sûre ne peut le confirmer.

Firth était avant tout un phonologue et un spécialiste de prosodie. Cette dernière qualité est à mon sens importante car elle explique sans doute la conception continuiste, non discrète, des faits de langue chez Firth. La collocation étant chez lui une propriété fondamentale du langage, une des grandes originalités de ce linguiste fut d'étendre les solidarités entre unités lexicales, aux dimensions plus abstraites des catégories grammaticales. Cette extension et son succès sont en fait assez paradoxaux puisque les seules lignes signées de Firth sur la grammaire ne dépassent pas quelques paragraphes. Je reprends ici ses propos, maintes fois cités ailleurs :

Collocations are actual words in habitual company. A word in a usual collocation stares you 


\section{LEGALLOIS}

in the face just as it is. Colligations cannot be of words as such. Colligations of grammatical categories related in a given structure do not necessarily follow word divisions or even subdivisions of words. (Firth 1957 : 14)

The statement of meaning at the grammatical level is in terms of word and sentence classes or of similar categories and of the inter-relation of those categories in colligation. Grammatical relations should not be regarded as relations between words as such - between « watched » and « him » in «I watched him»- but between a personal pronoun, first person singular nominative, the past tense of a transitive verb and the third person singular in the oblique or objective form. (Firth $1957: 13$ )

Comprendre la conception de la colligation chez Firth, est un exercice difficile; la dernière citation, qui constitue sans doute la seule pièce consistante, se révèle être assez déroutante : Firth semble décrire dans ses propos la combinaison entre catégories grammaticales dans la production d'une phrase. N'est-ce pas là, en fait, une définition minimale de ce qu'est la grammaire, plutôt qu'une perspective nouvelle sur des arrangements? En quoi l'exemple de Firth montrerait-il que les relations grammaticales seraient des associations d'un type semblable de celles qui valent pour les collocations? Le commentateur ne peut adopter qu'une attitude perplexe face à une conception et une illustration qui restera, chez Firth, particulièrement impressionniste. Telle est d'ailleurs l'attitude adoptée par T. Langendoen (1968) et M. Hoey (2005). Pourtant, malgré ce déficit, et sans faire véritablement florès, le terme est utilisé dans le milieu contextualiste britannique, c'est-à-dire chez ceux que l'on qualifie de néo-firthiens : par exemple, chez T.F. Mitchell dans une réflexion sur les verbes à particules / prépositions (T.F. Mitchell 1958); la colligation est alors chez ce linguiste une généralisation (au niveau des classes grammaticales) des collocations de mots. Ainsi : "heavy drinker » is an exemplification of the colligation «adjective + agentive noun » (Mitchell $1966: 337$ ). On reste ici, malgré tout, dans une identification de schémas 


\section{La colligation}

productifs - donc il s'agit là d'une « collocation grammaticale » façon Benson et al. (1995) ou Hausmann (1989). Il n'y a pas nécessairement attirance, pour reprendre l'exemple, entre la catégorie de l'adjectif et celle des noms agentifs. Halliday (1959) utilise le mot colligation dans son étude sur la langue chinoise : son sens exprime la relation entre un mot et une structure grammaticale. La notion hallidayenne de lexico-grammar est sans aucun doute une reformulation plus consolidée de la colligation.

Mais la colligation a reçu une certaine notoriété dans l'Ecole qui prolongea le contextualiste neofirthien : l'Ecole de linguistique de corpus de J. Sinclair ; Sinclair lui-même propose une définition :

Colligation is similar to collocation in that they both concern the cooccurrence of linguistic features in a text. Colligation is the occurrence of a grammatical class or structural pattern with another one, or with a word or phrase. "Negative", "possessive» and «modal» are the kinds of largely grammatical categories that figure in colligation. The term was first used by J. R. Firth, and has been widened a little for corpus work. (Sinclair 2003 : 145)

A partir de ces conceptions et des différents emplois passés ou actuels de colligation, je proposerais d'appréhender :

- la collocation comme un phénomène d'association entre mots lexicaux (un gros buveur), entre mots lexicaux et grammaticaux (un jour sans), entre mots grammaticaux (le * de * - le livre de Marie) ;

- la colligation comme un phénomène d'association entre un mot lexical ou grammatical et une catégorie grammaticale (partie du discours, fonction syntaxique, marqueurs aspectuels, modaux, temporels, marqueurs grammaticaux des catégories de la négation, de la propriété, etc.).

Les généralisations de structures à partir de collocations ne mènent donc pas à des «collocations grammaticales » mais à des schémas (productifs ou non), ou si on veut, à des constructions (par ex. V+Adv). On pourrait certes considérer que ces schémas très généraux sont constitués d'éléments en relation statistiquement significative : mais quel serait, alors, la 


\section{LEGALLOIS}

pertinence notionnelle de la colligation ? En français, un déterminant suit statistiquement plus souvent un nom qu'un adjectif ; un adjectif est le plus souvent associé à un nom. Au niveau fonctionnel, le complément d'objet est très généralement placé après le verbe. Il s'agirait alors d'affirmer que la colligation n'est juste qu'un autre nom pour grammaire. On le voit, l'apport serait extrêmement pauvre, même si on peut concevoir que les relations grammaticales sont les résultats «historiques» de diverses grammaticalisations, donc de diverses associations routinisées dans / par l'usage pour des raisons pragmatiques.

Il me semble donc préférable d'attribuer à la colligation une véritable originalité, donc de la considérer comme une corrélation étroite - statistiquement mesurable et pertinente - entre une forme lexicale et une catégorie fonctionnelle. L'examen de l'emploi de la locution à proprement parler me permet ici de récapituler les différentes catégories présentées.

Dans un semestre du journal Le Monde (2005), rubrique « International », on relève 47 occurrences (seulement!) de la locution : $80 \%$ de ces occurrences (38) sont employées à la forme négative : il y a donc :

- une collocation grammaticale forte avec les unités « ne...pas », eux même en relation associative très marquée ;

- une colligation avec l'expression de la négation.

De plus, 18 occurrences $(38 \%)$ sont construites à partir d'une forme attributive (N1 n'est pas à proprement parler Adj / $G N)$; on peut donc distinguer à nouveau une colligation marquée entre la locution et une structure syntaxique particulière.

La partie suivante, qui porte sur des études sur la colligation menée dans le contexte de la linguistique de corpus initiée par J. Sinclair, montre des extensions possibles du domaine colligationnel.

\subsection{Actualités de la colligation}

Sans entrer dans une présentation exhaustive, j'indiquerai ici, en fait, les directions que peut prendre une réflexion sur la colligation, à partir de trois exemples.

\subsubsection{G. Francis}

Premièrement, le travail de G. Francis (1991), déjà ancien et malheureusement peu suivi, qui propose de concevoir que le 


\section{La colligation}

système lexico-grammatical est intrinsèquement probabiliste : toute unité lexicale possède sa propre grammaire. On peut lier cette observation à celle de la théorie du lexique-grammaire en France, mais cette école n'a jamais véritablement validé sur corpus cette propriété.

En examinant, à partir du Cobuild Corpus, le comportement de quelques-uns des noms les plus fréquents en anglais, G. Francis détermine la fréquence des fonctions des syntagmes dont ces noms sont la tête. Je ne donne ici que l'exemple du nom impact.

Ce nom possède deux acceptions :

1 - l'effet que quelque chose a, sur une situation, un procès, une personne, etc.

2 - l'action d'un objet en contact avec un autre. Il est remarquable que les deux acceptions induisent des comportements syntaxiques différents. Ainsi, sur 100 occurrences, on identifie, en termes de fonctions grammaticales, la distribution suivante :

Tableau 2. Distribution du mot impact selon sa fonction grammaticale

\begin{tabular}{|l|c|c|c|c|c|}
\hline IMPACT & sujet & objet & Attribut & Adjoint & Total \\
\hline Acception 1 & 6 & 61 & 2 & 14 & 83 \\
\hline Acception 2 & 3 & 2 & 2 & 10 & 17 \\
\hline Total & 9 & 63 & 4 & 24 & 100 \\
\hline
\end{tabular}

Très nettement, l'acception 1 préfère les emplois en fonction « objet», et l'acception 2, dont les occurrences sont plus rares, la fonction « adjoint ». Ce comportement idiosyncrasique témoignerait donc d'un " déterminisme " grammatical, ou si on veut, d'une nature fondamentalement probabiliste du système lexico-grammatical. Un travail d'analyse sur un ensemble appréciable de noms pourrait confirmer que la colligation est au cœur d'un principe d'idiomaticité généralisé (Sinclair 1987).

\subsubsection{J. Sinclair}

Chez J. Sinclair, la colligation participe, avec d'autres propriétés phraséologiques, à la détermination de "l'unité lexicale étendue » : une séquence linguistique, possédant un « cœur lexical» et des caractéristiques syntaxique, sémantique et pragmatique. 


\section{LEGALLOIS}

Je reprends l'exemple ici de true feelings. Il existe pour ce cœur lexical :

- une collocation lexicale : l'expression est employée préférentiellement avec les verbes to hide/reveal/express;

- une collocation grammaticale: l'expression est déterminée préférentiellement par les possessifs his/their/yours ;

- une colligation : le cœur lexical est précédé par la structure «verbe + article possessif »; il est donc préférentiellement objet ;

- une préférence sémantique pour : 1) les verbes exprimant un procès de "manifestation 》 (montrer, cacher, exprimer...) ; 2) la possession (réalisée par les articles). Remarquons que la possession étant une dimension sémantique grammaticalement exprimée, ont peut considérer qu'il y a une autre colligation à l'œuvre ;

- une prosodie sémantique, c'est-à-dire une association avec une attitude pragmatique particulière : ici la réticence (reluctance) ou la difficulté, pour le sujet, de manifester ses « vrais sentiments ».

Ce complexe de propriétés doit être considéré comme un enrichissement majeur de la description lexicologique, voire lexicographique. La colligation y est un facteur important, qui montre à nouveau la nature probabiliste de l'usage des unités lexicales.

\subsubsection{Hoey}

Hoey (2005) a récemment fortement enrichi la notion de colligation, en l'appliquant à des phénomènes de tactique - au sens de positionnement. Hoey défend ainsi sa thèse du « lexical priming $»$ : tout mot est amorcé pour être employé en compagnie d'autres mots, dans certaines positions ou fonctions grammaticales, à un ou des endroits particuliers de la phrase, du paragraphe ou du texte. La colligation se définit comme suit :

a) la compagnie grammaticale qu'un mot ou qu'une séquence de mots privilégie ou évite ;

b) les fonctions grammaticales préférées ou évitées par le syntagme auquel appartient le mot ou la séquence de mots ;

c) la position dans une séquence (phrase, paragraphe, texte) qu'un mot ou séquence de mots préfère (ou évite). 


\section{La colligation}

Il ressort que selon la conception défendue plus haut: a) relève de la collocation, et b) de la colligation. L'originalité de Hoey est donc d'avoir introduit une dimension tactique (c) : la colligation textuelle. Ce dernier type, à bien y regarder, n'est jamais que «l'ancienne» collocation, au sens du Harris cité par l'OED, ou, plus près de nous, de Jespersen. La colligation textuelle peut être illustrée par l'analyse que propose Hoey de consequence dans deux locutions, in consequence et as a consequence:

- in consequence a une forte colligation avec le thème et donc avec la première position dans la phrase, et a une tendance forte à refuser les postmodifications, surtout en position initiale ;

- as a consequence possède un comportement différent: l'expression a une forte association avec le thème de la phrase, préfère donc la position initale, a une forte tendance à refuser une postmodification en position initiale, mais accepte très souvent d'être postmodifié en position finale.

Il s'agit donc là encore, de pouvoir mener des études plus développées pour apprécier la systématicité de l'amorçage lexical et de la colligation textuelle, à la fois, pour développer une analyse discursive qui conçoit les régularités constructionnelles des textes, mais aussi pour que la théorie linguistique en général soit mieux informée des pratiques de l'usage qui déterminent le système de la langue. D'ailleurs, dans les perspectives diachroniques qui visent la compréhension de la grammaticalisation et de la pragmaticalisation, la colligation textuelle est d'une importance fondamentale ; pour l'anglais, un même marqueur selon sa position initiale ou finale dans la phrase, a une tendance forte pour exprimer soit la subjectivité du locuteur (position initiale), soit l'interjubectivité (position finale).

La présentation succincte de ces quelques études indique que l'analyse de la colligation n'en est qu'à ses débuts, et qu'elle peut révéler des particularités de fonctionnements insoupçonnés, tant en synchronie qu'en diachronie.

\section{Des cadres collocationnels ou motifs}

Cette section s'intéresse à quelques « incarnations » des notions de collocation grammaticale et de colligation. Par « incarnation », 


\section{LEGALLOIS}

j'entends des unités particulières, détectables souvent uniquement grâce à des outils informatiques, de façon entièrement inductive, par une méthode «corpus-driven ». Je partirai donc des cadres collocationnels (collocational frameworks) de Renouf et Sinclair (1991), dont la complexification mène à ces unités désignées sous l'étiquette de motif.

\subsection{Les cadres collocationnels}

Les cadres collocationnels sont des combinaisons discontinues d'items grammaticaux qui encadrent des mots lexicaux : a + ?+ of ( a lot/number/kind/matter...of) ; be + ? + to (be able/allowed/ expected/made...to); for + ? of (for most/one/both/some...of); too $+?+$ to (too late/much/young/easy...to), etc.

Je ne prétends pas, bien sûr, que ces phénomènes de cadres aient été ignorés par la linguistique avant l'article de Renouf et Sinclair (par ex. Gaatone 1976). L'originalité des auteurs britanniques est de montrer à la fois la fréquence importante de ces cadres, et leurs associations avec des unités lexicales sémantiquement homogènes. Les cadres collocationnels sont des unités de langue, même s'ils ne sont pas habituellement mentionnés dans les descriptions linguistiques.

On peut voir également les cadres collocationnels comme une complexification de segments répétés (Salem 1987), ou encore comme une généralisation de ces segments; ainsi, récemment, Biber (2009) a-t-il mis en évidence pour le discours académique des patterns avec slots variables; par exemple, $[O n$ the * of, As a * of, In the * of, It is * that, etc.]. Ces cadres constituent à mon sens, un niveau intermédiaire entre des collocations grammaticales et des colligations. En effet, il est assez évident que «*» dans ces patterns, ne renvoie pas seulement à des unités lexicales différentes, mais à une classe lexicale précise (des noms, des adjectifs, etc.). La question se pose alors de la conception d'outils qui permettraient d'extraire des unités composées à la fois d'unités lexicales ou grammaticales et d'unités fonctionnelles. Il s'avère que certains linguistes ont réfléchi à ce type d'unités, et que, "de leur côté » des informaticiens ont élaborés des outils adéquats, pour des recherches a priori très différentes. Je présente donc, dans ce qui suit, la notion de motif. 


\section{La colligation}

\subsection{Les motifs}

Dans divers travaux (notamment Longrée, Luong \& Mellet 2008, Longrée \& Mellet, à par.), D. Longrée et S. Mellet ont insisté sur la pertinence d'un type d'unité textuelle, dont les caractéristiques sont une combinaison d'éléments lexicaux et grammaticaux. Dans les textes observés, ces unités, appelées motifs, ont souvent une fonction d'organisateur du discours, ou une valeur argumentative :

De manière strictement formelle, un motif se définit par l'association récurrente de $\mathrm{n}$ éléments du texte muni de sa structure linéaire (Legallois 2006), laquelle donne une pertinence aux relations de successivité et de contiguité (Longrée, Luong \& Mellet 2008 ; Mellet \& Longrée 2009). Ainsi, si le texte est formé d'un certain nombre d'occurrences des éléments A, B, C, D, E, un motif pourra être la micro-structure récurrente $\mathrm{ACD}$ ou bien encore AA, etc., sans qu'on préjuge ici de la nature des éléments $\mathrm{A}, \mathrm{B}, \mathrm{C}, \mathrm{D}, \mathrm{E}$ en question. En effet, la notion de motif est conçue comme un moyen de conceptualiser la multidimensionalité (ou le caractère multi-niveau) de certaines formes récurrentes qui sollicitent à la fois le lexique, les catégories grammaticales et la syntaxe, éventuellement la prosodie, la métrique (Longrée \& Mellet, à par.).

Le motif est donc une unité multidimensionnelle, c'est-à-dire constituée à la fois d'associations lexicales ou grammaticales, d'appariements entre forme et sens ou fonction pragmatique / discursive, entre forme et fonction grammaticale. Son analyse pose encore des problèmes, car sa détection automatique demande un outillage complexe. Néanmoins, il est possible de donner quelques exemples de motifs relativement simples.

Ainsi, dans la perspective de contraster des genres différents, j'ai étudié les séquences répétées spécifiques de deux corpus (non lemmatisés): des débats parlementaires à l'Assemblée Nationale et un ensemble d'ouvrages contemporains de philosophie (essentiellement Derrida, Foucault et Onfray). Certaines de ces séquences répétées peuvent être généralisées ; autrement dit, on peut distinguer un patron abstrait 


\section{LEGALLOIS}

qui subsume différents types d'occurrences. Ce patron constitue un motif. Je ne donne ici que deux motifs représentatifs : pour le genre "débats à l'Assemblée Nationale », le motif «les plus Adj» est particulièrement sur-représenté : les plus fragiles, modestes, jeunes, démunis, faibles, pauvres, défavorisés, vulnérables, nantis etc. Par exemple:

(1) Monsieur le ministre, vous avez parlé de l'enrichissement de nos débats, pour ce qui est de votre projet, il tend à enrichir les plus riches, comme M. François Pinault.

(2) Concentrer son action sur la baisse de la fiscalité, c'est oublier les plus défavorisés.

La structure permet de désigner une classe sociologique de personnes, constituant souvent l'enjeu, dans les discussions parlementaires, d'une confrontation avec une autre classe opposée. «Les plus Adj» est un motif simple: il englobe certains 3-grams spécifiques, donc statistiquement saillants (par ex. : les plus défavorisés), mais aussi d'autres séquences qui peuvent être des hapax: les plus malchanceux, les plus repus, par exemple. Ces hapax répondent donc au même modèle particulièrement caractéristique du débat parlementaire.

Pour le discours philosophique, je retiens le motif «N1 comme $\mathrm{N} 2$ de $\mathrm{N} 3$ » dont la fonction est de construire une relation d'identité entre deux concepts, ou plus exactement, d'exprimer que N1 doit être envisagé sous l'aspect du GN « N2 de $\mathrm{N} 3 »$; par exemple :

(3) La question de l'être, comme question de la possibilité du concept (Derrida, L'écriture et la Différence)

(4) l'extériorité comme essence de l'être (Derrida, L'écriture et la Différence)

(5) l'inconscient comme signification de nos conduites (Foucault, Dits et Inédits)

(6) la révolution comme condition de réalisation de la jouissance (Onfray, Anti-manuel de philosophie)

Ces deux motifs sont donc multidimensionnels en tant qu'ils combinent mots et catégories, collocation et colligation. Ils représentent des phrases (au sens de "manières de parler »les phrases de la phraséologie) de discours spécifiques, des 
signatures de la dimension générique des textes. Ils ne peuvent être directement extraits d'une recherche de segments répétés, mais construits après une généralisation de ce type de segments. Cette généralisation est possible quant on s'intéresse à des segments de longueur déterminée (par exemple, 4-grams), et en considérant que la ou les variables (dans les exemples, les catégories grammaticales) remplissent des «slots » définis; la généralisation devient plus problématique si on considère que les variables ne sont pas encadrées par les unités invariables dans un empan fixe (ici de 1), mais dans un empan plus élargicomme proposé dans la définition de D. Longrée et de S. Mellet plus haut. Il convient donc de rechercher des outils à partir desquels des motifs complexes (c'est-à-dire non limités par des empans, prenant en compte plusieurs dimensions à la fois) pourraient être directement identifiés.

Dans une perspective de fouille de textes pour l'extraction, dans les bases de données, de chaînes d'ADN ou de séquences de protéines, le laboratoire Greyc de Caen a élaboré un algorithme qui permet d'identifier les motifs multidimensionnels, selon le principe suivant :

On dit qu'une séquence $\mathrm{S} 1$ est incluse dans une séquence $\mathrm{S} 2$ (S1 est une sous-séquence de S2) si on retrouve dans S2 tous les éléments de $\mathrm{S} 1$ dans le même ordre : par exemple $<$ b,e $>$ est une sous-séquence de la séquence $<\mathrm{a}, \mathrm{b}, \mathrm{c}, \mathrm{d}, \mathrm{e}>$ mais $<\mathrm{e}, \mathrm{b}>$ ne l'est pas.

Dans le cadre d'une collaboration avec mes collègues du Greyc (dont Th. Charnois), quelques expérimentations ont été amorcées, qui déjà, donnent des résultats assez encourageants, malgré certaines difficultés méthodologiques. Toujours dans une perspective d'analyse générique, nous avons calculé les motifs émergents du genre poétique $\left(19^{\mathrm{e}}\right.$ siècle) par rapport au genre romanesque $\left(19^{\mathrm{e}}\right.$ siècle) et au genre correspondance ( $19^{\mathrm{e}}$ siècle). A nouveau, je ne présente que quelques résultats. Voici les patrons qui semblent les plus intéressants :

\section{qu'on V1 et qu'on V2}

(7) comme un front de coursier qu'on flatte et qu'on caresse (Lamartine, Jocelyn) 


\section{LEGALLOIS}

(8) L'amour vaut mieux, au fond des antres frais,

Que ces soleils qu'on ignore et qu'on nomme (Hugo, Les Contemplations)

\section{des $N$ plus Adj que}

(9) il sait des secrets plus vieux que la tombe (Dierx, Les Lèvres closes)

(10) si la lame le ballotte, comme une épave, pendant des heures plus prolongées que la vie d'homme (Lautréamont, Les Chants de Maldoror)

\section{le N1 qui V et le N2 qui $\mathrm{V}$}

(11) Penché, l'oeil immobile, il observe avec joie le liége qui s'enfonce et le roseau qui ploie (Delille, L'Homme des champs ou les Géorgiques françaises)

(12) le coeur qui se repose et le sang qui s'affaisse (Verlaine, Oeuvres poétiques complètes)

\section{le N1 du N2 qui V dans}

(13) C'est le puits du néant même

Qui s'ouvre dans ce zéro (Hugo, Les Chansons des rues et des bois)

(14) On entend le bruit du vieux qui bêche dans la nuit (Bouilhet Dernières chansons)

(15) L'haleine du zéphyr qui dans l'air se balance (Baour-Lormian, Ossian)

Ces motifs constituent des combinaisons de mots grammaticaux et de catégories grammaticales. D'une certaine manière, ils combinent collocation et colligation, même si ici, la colligation se déduit logiquement de la collocation (par ex. l'emploi des articles devant un nom). Surtout, ils constituent une phraséologie propre au genre : cadres pré-déterminés, lexico-grammaticaux, mais aussi prosodiques et rythmiques : ils fonctionnent comme des tuteurs d'unités rythmiques. Ce sont des stylèmes, ne présentant pas d'ailleurs toujours la même structure; on le remarque pour (12), (13) et (14) : c'est N2 qui est expansé par la relative en (14), alors que c'est N1 en (13) et (15). (15) se démarque de (13) et (14) par la permutation de V avec le SP. Les motifs acceptent donc des « déformations », c'est d'ailleurs 


\section{La colligation}

leur plasticité qui constitue leur pertinence fonctionnelle, mais qui empêche aussi qu'ils soient aisément détectables.

Le motif «le N1 du N2 qui V dans», une fois reprojeté sur les textes, permet de saisir des phénomènes intertextuels qu'une analyse exclusivement lexicale peut peut-être pointer, mais sans mettre en relief la spécificité syntagmatique de leur mise en discours. Considérons ces vers :

(16) le vent du soir qui meurt dans le feuillage (Baour-Lormian, Ossian, 1827)

(17) la brise du soir qui pleure dans des branches de coudrier (Sainte-Beuve, Poésies, 1829)

(18) la voix du torrent, qui gémit et se brise dans l'abîme en sanglots (Lamartine, Harmonies poétiques et religieuses, 1830)

(19) le bruit du fleuve entier qui tombe dans un abîme (Lamartine, Harmonies poétiques et religieuses, 1830)

(20) le vent du désert qui souffle dans mes cheveux (Quinet, Ahasvérus, 1833)

(21) le grand bruit du rêveur océan qui parle dans la nuit (Guérin, Poèmes, 1839)

(22) le chant des brises qui sifflent dans la lande (Brizeux, Marie, 1840)

(23) Du vent qui s'endort dans les bois! (Hugo, Les Orientales, 1840)

(24) L'ange du soir rêveur, qui flotte dans les vents (Hugo, Les Contemplations, 1856)

(25) La feuille des forêts qui tourne dans la bise (Moréas, Les Syrtes, 1884)

Chez huit auteurs différents, des motifs très proches émergent, exprimés par les isotopies lexicales suivantes : vent \{vent, brise, bise $\}$, nuit \{nuit, soir\}, mort \{mourir, gémir, pleurer, tomber, se briser, abîme\}, feuillage \{feuillage, feuille, branches, bois\}. On remarquera que (25) exprime une relation actancielle exactement inverse de (16). Les thèmes romantiques sont évidemment présents dans ces vers, du reste la projection du même patron syntaxique sur des corpus poétiques de périodes 


\section{LEGALLOIS}

différentes $\left(18^{\mathrm{e}}\right.$ ou $20^{\mathrm{e}}$ siècle $)$ ne permet pas d'observer cette même homogénéité.

A la lumière de cet exemple, on peut penser que les motifs sont de formidables points d'entrée dans les textes, pour observer des relations intertextuelles ou thématiques dont les formes sont elles-mêmes des reprises de manières plus ou moins fixées (donc mémorisables) pour dire l'expérience.

\section{Conclusion}

Mon objectif a donc été de proposer un panorama des perspectives - anciennes ou nouvelles - qui examinent l'implication des éléments grammaticaux (les « struments ») dans les relations d'association mutuelle. S'intéresser plus particulièrement à la dimension grammaticale des collocations n'a pas pour objectif d'accentuer le clivage entre éléments grammaticaux et éléments lexicaux ; au contraire, il s'agit bien de montrer que ces deux catégories possèdent des caractéristiques proches en matière de collocation. Le concept de colligation, encore relativement original, me semble être à distinguer de la "simple " collocation grammaticale: elle porte sur les phénomènes de préférences régulières entre les unités lexicales ou grammaticales et 1) les fonctions ou catégories syntaxiques, 2) l'expression d'une catégorie sémantique grammaticalisée (modalité, aspect, négation...), 3 ) les positions dans la proposition, voire dans le texte. Ainsi, l'analyse colligationnelle intéresse aussi bien les recherches sur les changements syntaxiques, sur la grammaticalisation, que sur l'organisation textuelle.

Dans l'ensemble des incarnations de la collocation grammaticale / colligation, l'unité « motif» est la plus récente ; encore difficilement détectable par une démarche inductive, elle devient peu à peu appréhendable par des outils issus de la fouille de données. J'ai voulu donner ici quelques illustrations de ce que ce type de recherche apporte, par rapport, notamment, à une analyse de segments répétés. Beaucoup - énormément reste à faire dans ce domaine, puisque le chantier est encore à peine ouvert. 


\section{La colligation}

\section{Références bibliographiques}

Bally Ch. (1909). Traité de stylistique française, 1 et 2. Genève : Atar.

Bartsch S. (2004). Structural and Functional Properties of Collocations in English. A Corpus Study of Lexical and Pragmatic Constraints on Lexical Cooccurrence. Tübingen : Narr.

Benson M., Benson E. \& Ilson R. (1986). Lexicographic Description of English. Amsterdam/Philadelphia : John Benjamins.

Firth J.R. (1957). « A synopsis of linguistic theory 1930-1955 », in Studies in Linguistic Analysis. Oxford: Philological Society, 1-32.

Francis G. (1991). « Nominal group heads and clause structure », Word 42, 2 : 144-156.

Gaatone D. (1976). «Locutions prépositives et groupes prépositionnels », Linguistics $167:$ 15-33.

Gledhill C. \& Frath P. (2007). «Collocation, phrasème, dénomination : vers une théorie de la créativité phraséologique», La Linguistique 43(1) : 65-90.

Granger S. \& Paquot M. (2008). «Disentangling the phraseological web », in Granger S. \& Meunier F. (eds), Phraseology: An interdisciplinary perspective. Amsterdam/ Philadelphia : John Benjamins, 27-49.

Halliday M. A. K. (1959). The language of the Chinese 'Secret history of the Mongols'. Oxford: Basil Blackwell for the Society (Publications of the Philological Society, XVII).

Hausmann F. J. (1989). «Le dictionnaire de collocations », in Hausmann F.J., Reichmann O., Wiegand H.E. \& Zgusta L. (eds), Wörterbücher: ein internationales Handbuch zur Lexicographie. Dictionaries. Dictionnaires. Berlin/ New York : De Gruyter, 1010-1019.

Heid U. (1994). « On Ways Words Work Together - Topics in Lexical Combinatorics », Euralex 94 Proceedings, Vrije Universiteit Amsterdam, 226-257. 


\section{LEGALLOIS}

Jespersen O. (1917). Negation in English and other languages. Copenhagen: Publisher Kgl. Danske videnskabernes selskab.

Jespersen O. (1922). Language, its nature, development, and origin. New York : H. Holt.

Langendoen D. T. (1968). The London School of Linguistics : A Study of the Linguistic Contributions of B. Malinowski and J. R. Firth. Cambridge, MA : MIT.

Legallois D. (2006). «Des phrases entre elles à l'unité réticulaire de textes », Langages $163: 56-70$.

Legallois D. (à paraître). «From grammaticalization to expressive constructions : the case of histoire de + inf. ", in Bouveret M. \& Legallois D. (eds), Constructions in French, Amsterdam / Philadelphia : Benjamins.

Léon J. (2008). « Aux sources de la 'Corpus Linguistics' : Firth et la London School », Langages 171:12-33.

Longrée D. \& Mellet S. (à paraître). "Le motif: une unité phraséologique englobante? Etendre le champ de la phraséologie de la langue au discours », Langages (D. Legallois \& A. Tutin, coord.).

Longrée D., Luong X. \& Mellet S. (2008). «Les motifs : un outil pour la caractérisation topologique des textes », in S. Heiden \& B. Pincemin (éd.), JADT 2008, Actes des 9èmes Journées internationales d'Analyse statistique des Données Textuelles, vol. 2. Lyon : Presses universitaires de Lyon, 733-744. Consultable en ligne à l'adresse : http://lexicometrica.univ-paris3.fr/jadt/jadt2008/pdf/longreeluong-mellet.pdf.

Magri V. \& Purnelle G. (2012). « Mot à mot, brin par brin: les suites [Nom préposition Nom] comme motifs », in JADT 2012, Actes des 11èmes Journées internationales d'Analyse statistique des Données Textuelles. Consultable en ligne à l'adresse: lexicometrica.univ-paris3.fr/jadt/ jadt2012/Communications/Magri, Veronique et al. - Mot a mot, brin par brin.pdf. 


\section{La colligation}

Mellet S. \& Longrée D. (2009). « Syntactical Motifs and Textual Structures », Belgian Journal of Linguistics 23 (New Approaches in Textual Linguistics) : 161-173.

Mitchell T. F. (1958). "Syntagmatic relations in Linguistic Analysis ", Transactions of the Philological Society 56 : 101-118.

Mitchell T. F. (1971). "Linguistic "goings on"; collocations and other lexical matters arising on the syntactic record", Archivum Linguisticum, v. 2.

Palmer H. E. (1933). Second Interim Report on English Collocations. Tokyo : Kaitakusha.

Pottier B. (1968). « La grammaire générative et la linguistique », Travaux de linguistique et de littérature VI(1) : 7-25.

Quiniou S., Cellier P., Charnois Th. \& Legallois D. (sous presse). «What About Sequential Data Mining Techniques to Identify Linguistic Patterns for Stylistics?», in Lecture Notes in Computer Science.

Rebori V. (2002). «The legacy of J.R. Firth : a report on recent research », Historiographica Linguistica XXIX(1/2) : 165190.

Renouf A. \& Sinclair J. (1991). "Collocational frameworks in English », in K. Aijmer \& B. Altenberg (eds), English Corpus Linguisitcs. Studies in honour of Jan Svartvik. London : Longman, 128-144.

Salem A. (1987). Pratique des segments répétés. Essai de statistique textuelle. Paris, Klincksieck.

Sinclair J. (2003). Reading Concordances. An Introduction. London : Longman.

Sinclair J. (1987). " The nature of the evidence », in J. McH. Sinclair (ed.), Looking Up : An Account of the COBUILD Project in Lexical Computing. London : Collins.

Traugott E.C. (à paraître). «On the function of the espitemic adverbs surely and no doubt at the left and right periphery of the clausen ", in K. Beeching \& U. Detges (eds), The role of left and rightperiphery in semantic change. Amsterdam : Benjamins. 


\section{LEGALLOIS}

Tutin A \& Grossmann F. (2002). «Collocations régulières et irrégulières: esquisse de typologie du phénomène collocatif», Revue française de Linguistique appliquée, Lexique : recherches actuelles, vol. VII(1) : 7-25.

Tutin A (à paraître). «Les collocations lexicales : une relation essentiellement binaire définie par la relation prédicatargument », Langages (D. Legallois \& A. Tutin, coord.). 